\title{
Saphenofemoral Complex: Anatomical Variations and Clinical Significance
}

\author{
Ehab Mostafa Elzawawy*, Ayman Ahmed Khanfour \\ Anatomy and Embryology Department, Faculty of Medicine, University of Alexandria, Alexandria, Egypt
}

Email address:

ehabzawawy@gmail.com (E. M. Elzawawy)

${ }^{*}$ Corresponding author

\section{To cite this article:}

Ehab Mostafa Elzawawy, Ayman Ahmed Khanfour. Saphenofemoral Complex: Anatomical Variations and Clinical Significance. International Journal of Clinical and Developmental Anatomy. Vol. 4, No. 1, 2018, pp. 32-39. doi: 10.11648/j.ijcda.20180401.15

Received: July 13, 2018; Accepted: August 10, 2018; Published: September 5, 2018

\begin{abstract}
Varicosities of great saphenous vein (gsv) or its tributaries are a common medical condition present in up to $25 \%$ of adults. The gsv and its tributaries are located in a fascial compartment on the front of the thigh. There are great anatomical variations of these veins. However, the relation between these veins and the fascia lata on the front of thigh is even more variable and carries greater clinical importance. Forty cadaveric lower limbs were dissected to examine anatomical variations of these veins and describe their relation to the deep fascia of the thigh. Fascia lata of the front of the thigh split into superficial saphenous fascia and deep fascia lata proper. This fascial splitting formed the saphenous compartment. There were 3 types of saphenous compartment. Type $1(30 \%)$, there was a triangular saphenous compartment containing the gsv and its tributaries. Type $2(30 \%)$, there was a fascial canal containing the gsv. Type $3(40 \%)$, there was a small fascial saphenous compartment with variable boundaries that contained the gsv and 1 or 2 of its tributaries. The number of superficial tributaries in the front of the thigh ranged from 3-7 with a mean of 5.12 \pm 1.95 . The length of the gsv in the saphenous compartment ranged from 5-8.5 $\mathrm{cm}$ with a mean of $6.43 \pm 1.65 \mathrm{~cm}$. The length of tributaries in the saphenous compartment ranged from $2-6 \mathrm{~cm}$ with a mean of $3.82 \pm 2.74 \mathrm{~cm}$. The external pudendal artery (epa) was intimately related to saphenofemoral junction (sfj) in $30 \%$ of cases. The cutaneous branches of the femoral nerve were related to the gsv, its tributaries and sfj in $52.5 \%$ of cases. Precise identification of the location of the gsv, its tributaries in relation to deep fascia of the thigh, epa and cutaneous nerves is crucial for planning appropriate surgical technique in case of varicosities.
\end{abstract}

Keywords: Great Saphenous Vein, Superficial Tributaries, Fascia Lata, Saphenous Triangle

\section{Introduction}

Textbooks $[1,2]$ describe 6 gsv tributaries in the thigh; the posteromedial vein, the anterolateral vein and 4 peri-inguinal veins namely, superficial epigastric, superficial circumflex iliac and superficial and deep external pudendal. Most of these tributaries join the gsv, few of them join the common femoral vein (cfv). Collectively, all the tributaries are called sfj tributaries.

Souroullas et al [3] reported that superficial venous anatomy of gsv, its tributaries and $\mathrm{sfj}$ is highly variable. Donnelly et al [4] stated that these veins are so variable that they have no specific patterns.

The deep fascia of the thigh is fascia lata. The gsv passes through the saphenous opening in fascia lata to join the femoral vein (fv) to form the cfv. The cribriform fascia is the anatomical structure covering the saphenous opening. This fascia is pierced by gsv and by efferent lymphatics from superficial inguinal lymph nodes. Fascia lata at the saphenous opening displays superficial and deep strata [5]. The relation of the gsv, its tributaries and sfj to the fascia of the thigh is very important, but scarcely studied.

The proximal part of gsv is easily identifiable in ultrasonography due to its typical appearance known as the saphenous eye or Egyptian eye. The saphenous eye shows that the gsv is located within the saphenous compartment outlined by the superficial saphenous and deep aponeurotic layers of fascia lata [6]. 
The epa may be in close proximity to the gsv, its tributaries or the sfj. The artery may be severed during varicose vein surgery causing intra-operative or post-operative complications [4].

Standard varicose vein surgery is ligation of $\mathrm{sfj}$ accompanied by gsv stripping. It is considered the gold standard for varicose veins treatment [7].

Recently, new less invasive methods were introduced, including ultrasound-guided foam sclerotherapy, radiofrequency ablation and endovenous laser treatment. All these modalities necessitate that the operator must be familiar with the anatomical variations of the sfj to increase success and decrease recurrence rates [8].

The aim of this work was to study the anatomical variations of the gsv, its tributaries and the sfj and their relations to fascia lata, epa and cutaneous nerves to understand pathogenesis of varicosities and plan surgical procedures for varicose veins with better results and less complications.

\section{Material \& Methods}

\subsection{Material}

A total of 40 lower limbs from 20 cadavers (14 males and 6 females) were included in this study. All lower limbs were apparently normal with no apparent varicosities. All cadavers were fixed in $8 \%$ formalin and preserved in $30 \%$ ethanol.

\subsection{Method}

The fascia lata on the front of thigh was dissected to identify its layers. The number of the superficial tributaries at the $\mathrm{sfj}$, their location in relation to the layers of fascia lata and whether they terminated into the gsv or cfv were recorded. The relationship between all these veins and the epa and the cutaneous nerves of the front of the thigh was studied. All measurements were taken using Vernier caliper.

\subsection{Statistical Analysis}

All data was expressed as mean \pm standard deviation (SD) using Statistical Package for Social Sciences (SPSS/version 20) software [9].

\section{Results}

\subsection{Fascial Saphenous Compartment}

The fascia lata split into a superficial layer (saphenous fascia) and a deep layer (fascia lata proper) just below inguinal ligament thus creating a fascial space (saphenous compartment). There were 3 types of saphenous compartment. Type 1 (in $30 \%$ of cases), the space was triangular in shape with its base upwards at the inguinal ligament and its apex downwards. The gsv entered the front of the thigh through the medial border of the triangle just above its apex "Figures 1-4".

The triangular saphenous compartment is a closed fascial space except where the gsv entered near the apex and the tributaries entered at the sides "Figure 4". The cribriform fascia is a thin sheet of sieve like membrane covering the saphenous opening. It lied inside the compartment deep to saphenous fascia and superficial to fascia lata proper "Figure 1 ”.

The fascia lata over adductor longus muscle split at the medial border of this muscle. This line of splitting formed the medial border of the saphenous triangle. This border extended for a distance of $12.63 \pm 1.34 \mathrm{~cm}$. The fascia lata over sartorius muscle split at the medial border of this muscle. This line formed the lateral border of the triangle. It extended for a distance of $12.33 \pm 1.86 \mathrm{~cm}$. The base of the triangle was at the inguinal ligament and extended for $9.88 \pm 0.79 \mathrm{~cm}$ "Figures 4, 5" "Table 1". So, the boundaries of the saphenous triangle corresponded to the boundaries of the femoral triangle "Figure 4".

Type 2 (in $30 \%$ of cases), the fascial saphenous compartment was just a canal containing the gsv while all its tributaries were outside the fascial compartment "Figure 6". Type 3 (in $40 \%$ of cases), there was a small fascial saphenous compartment with variable boundaries that contained the gsv and 1 or 2 of its tributaries "Figures 7-10".

The thickness of saphenous fascia was very variable. It varied from 2-6 mm with a mean of $3.62 \pm 2.32 \mathrm{~mm}$. It was thin in men due to small fat content "Figures 1-4" and thick in women due to large content of superficial fat "Figure 5".

The gsv was in the fascial saphenous compartment in all cases, while its tributaries were superficial to the saphenous fascia and pierced it at variable distances before joining the gsv or cfv. The length of the gsv in the compartment ranged from $5-8.5 \mathrm{~cm}$ with a mean of $6.43 \pm 1.65 \mathrm{~cm}$. The length of tributaries in the saphenous compartment ranged from $2-6 \mathrm{~cm}$ with a mean of $3.82 \pm 2.74 \mathrm{~cm}$.

In type 1 , the tributaries pierced the saphenous fascia very early and ran most of their course inside the triangular saphenous compartment "Figures 1-5". In type 2 cases with gsv canal, tributaries were outside the fascial canal for their entire length "Figure 6". In type 3, most of tributaries pierced the saphenous fascia late in their course and ran a small distance inside the fascial compartment just before joining gsv "Figures 7-10".

\subsection{Variations of Veins}

In this study, all sfj tributaries that joined the axial veins directly (either gsv or cfv) were considered "Figures 1-5". Veins that coalesced then drained into the axial veins were considered as a single tributary "Figures 6, 7". Tributaries mainly terminated in gsv "Figures 1-9", while few terminated into the cfv "Figure 10" "Table 2". The fv and cfv were deep to fascia lata proper in the femoral sheath in all cases "Figures 7, 9".

The number of superficial tributaries joining sfj ranged from 3-7 with a mean of $5.12 \pm 1.95$ "Figures $1-10$ ". There were 3 tributaries in 16 cases (40\%) "Figure 6". There were 4 tributaries in 12 cases $(30 \%)$ "Figure 8 ". There 
were five tributaries in 9 cases $(22.5 \%)$ "Figure 5 ". In 2 cases $(5 \%)$, there were 6 tributaries "Figures $1-4$ ". In one cases $(2.5 \%)$, there were 7 tributaries "Figure 7 ". The most consistent gsv tributary was the superficial external pudendal vein, which was identified in 36 cases $(90 \%)$ "Figures 1-9". The superficial circumflex iliac was identified in 32 cases (80\%) "Figures 1-9", while the superficial epigastric vein was found in 30 cases $(75 \%)$ "Figures 1-4, 6-9". The tributaries draining directly into the $\mathrm{cfv}$ ranged from $0-1$ and were found in 12 cases $(30 \%)$ with 28 cases $(70 \%)$ having no tributaries draining into the cfv. The commonest tributary draining directly into the cfv was the superficial circumflex iliac vein in 10 cases $(25 \%)$ "Figure 10".

Bifid gsv was found in 3 cases $(7.5 \%)$, the distal part outside saphenous compartment could be bifid "Figure 2", or the proximal part inside saphenous compartment was bifid "Figure 10".

The epa was related to sfj in 12 cases $(30 \%)$. It crossed anterior to $\mathrm{sfj}$ in $10 \%$ and posterior to it in $15 \%$ of cases. It passed behind the anterolateral thigh vein and in front of gsv just below the sfj in one case $(2.5 \%)$ "Figure 9". In another case $(2.5 \%)$, it passed between the 2 limbs of a bifid gsv "Figure 10".

The cutaneous branches of the femoral nerve were found in the saphenous compartment in close proximity to gsv, sfj or tributary veins in $52.5 \%$ of cases "Figures 6,10 ".

Table 1. Dimensions of the saphenous triangle.

\begin{tabular}{llll}
\hline \multirow{2}{*}{ Length (cm) } & Dimension & & \\
\cline { 2 - 4 } & Medial border & Lateral border & Base \\
\hline Range & $11.2-14.1$ & $10.5-13.9$ & $9-10.5$ \\
Mean & 12.63 & 12.33 & 9.88 \\
Std. Deviation & 1.34 & 1.86 & 0.79 \\
\hline
\end{tabular}

Table 2. Number of superficial tributaries according to their termination.

\begin{tabular}{llll}
\hline $\begin{array}{l}\text { Number of } \\
\text { tributaries }\end{array}$ & \multicolumn{2}{l}{ Termination } & \\
\cline { 2 - 4 } & Into gs vein & Into cfv & Total \\
\hline Range & $3-7$ & $0-1$ & $3-7$ \\
Mean & 4.66 & 0.56 & 5.12 \\
Std. Deviation & 1.282 & 0.392 & 1.95 \\
\hline
\end{tabular}

Table 3. Comparison between studies regarding number of sfj tributaries.

\begin{tabular}{lllll}
\hline $\begin{array}{l}\text { Number of } \\
\text { tributaries }\end{array}$ & $\begin{array}{l}\text { Souroullas } \\
\text { et al [3] }\end{array}$ & $\begin{array}{l}\text { Donnelly } \\
\text { et al [4] }\end{array}$ & $\begin{array}{l}\text { Hemmati } \\
\text { et al [11] }\end{array}$ & $\begin{array}{l}\text { Present } \\
\text { study }\end{array}$ \\
\hline No tributaries & $1.2 \%$ & $0.4 \%$ & - & - \\
3 or less & $49.4 \%$ & $57.4 \%$ & $34.7 \%$ & $40 \%$ \\
$4-5$ & $47.1 \%$ & $38.1 \%$ & $60.1 \%$ & $52.5 \%$ \\
$\geq 6$ & $3.5 \%$ & $4.5 \%$ & $5.2 \%$ & $7.5 \%$ \\
Range & $0-7$ & $0-10$ & $2-7$ & $3-7$ \\
\hline
\end{tabular}

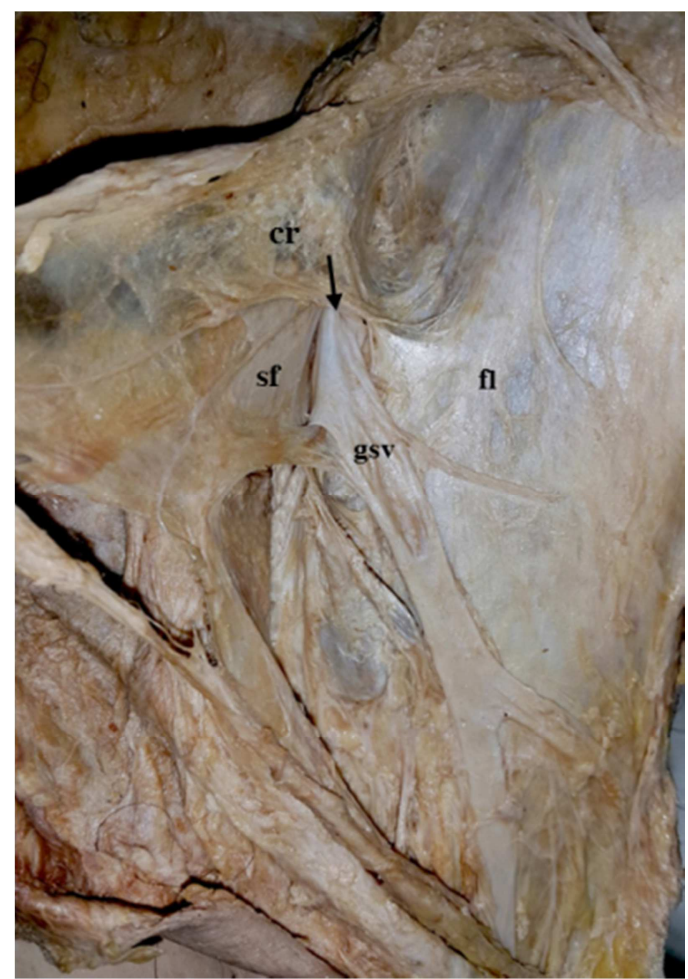

Figure 1. A photograph of right thigh. The saphenous fascia (sf) is dissected and reflected upwards and laterally. The gsv passes through the saphenous opening (arrow) in the cribriform fascia (cr). Fascia lata (fl) is seen deep to gsv.

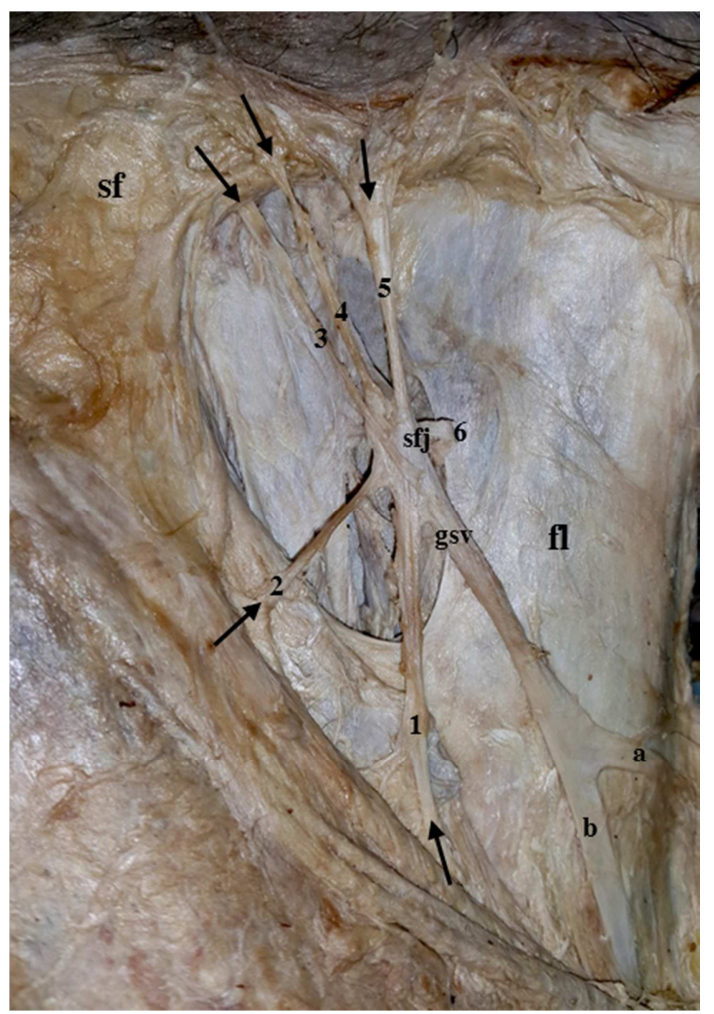

Figure 2. A photograph of the previous specimen after dissection of cribriform fascia. The gsv is bifid $(a, b)$. The sfj is joined by 6 tributaries (16). Fascia lata (fl) and saphenous fascia (sf) are noted. The tributaries pierce sf (arrows) to enter saphenous triangle and join the sfj. The superficial circumflex iliac is (3), superficial epigastric is (5) while superficial external pudendal is (6). 


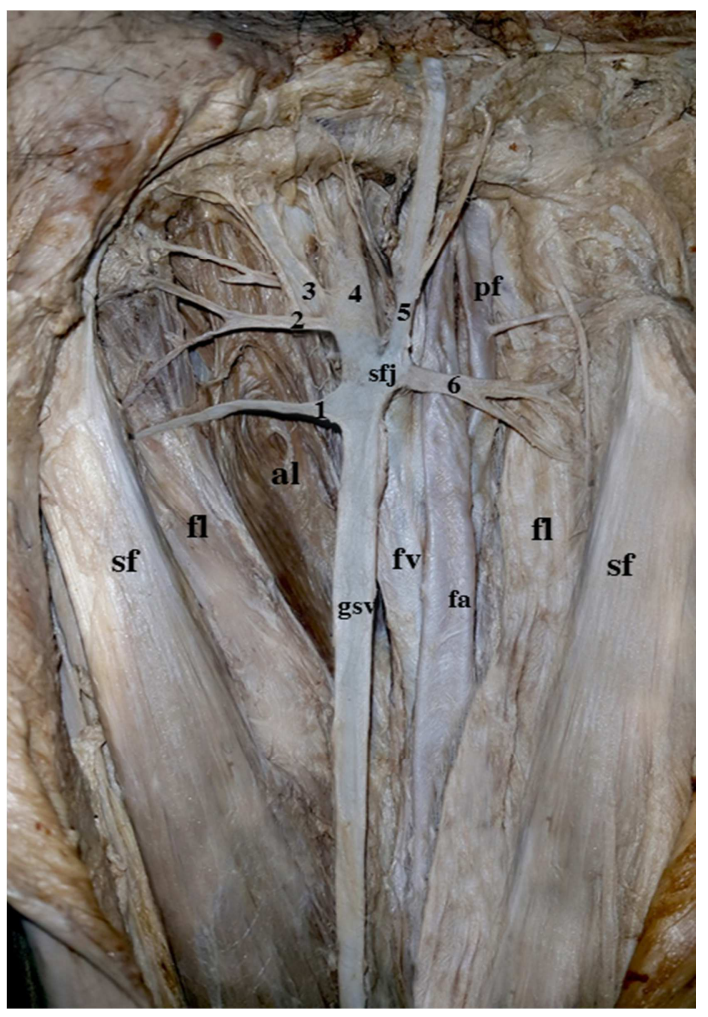

Figure 3. A photograph of left thigh. The gsv passes between saphenous fascia (sf) and fascia lata (fl). It is joined by veins (1-6) before it joins the femoral vein (fv) at the sfj. The femoral artery (fa), profunda femoris artery (pf) and adductor longus muscle (al) are noted. The superficial circumflex iliac is (5), superficial epigastric is (4) while superficial external pudendal is (1).

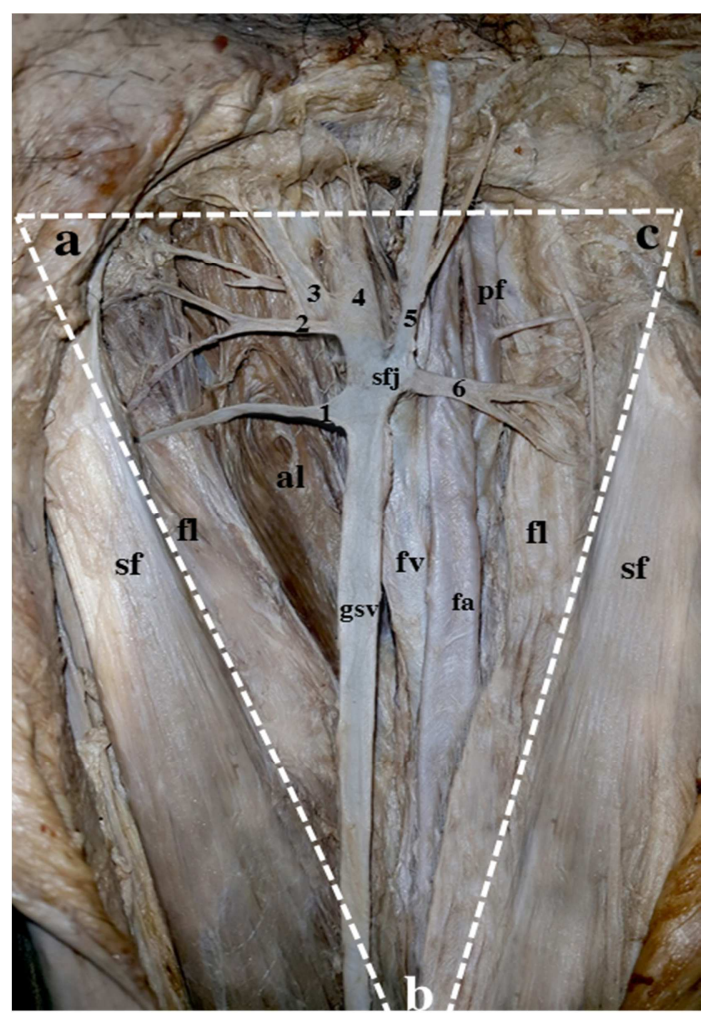

Figure 4. A photograph of the previous specimen showing the boundaries of the saphenous triangle. The medial boundary is ab, the lateral boundary is $b c$ and the base is ac.

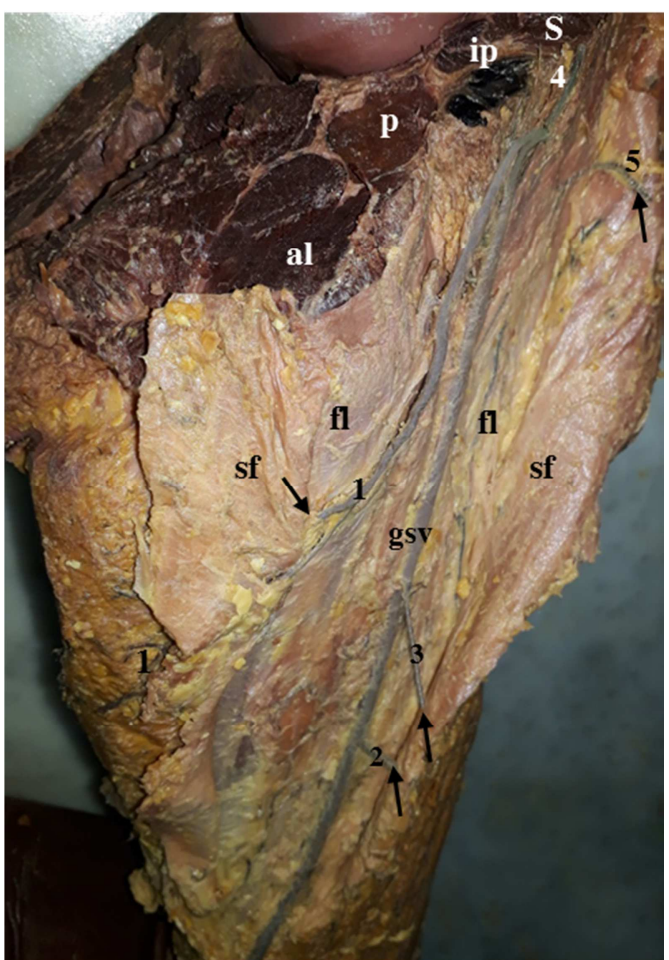

Figure 5. A photograph of left thigh of a female cadaver. The deep fascia of the front of thigh splits into superficial saphenous fascia (sf) and deep fascia lata proper (fl). The gsv is joined by 5 superficial tributaries (1-5). These tributaries pierce sf (arrows) to join gsv. Adductor longus (al), pectineus (p), iliopsoas (ip) and sartorius (s) muscles are noted. The superficial circumflex iliac is (4), while superficial external pudendal is (1).

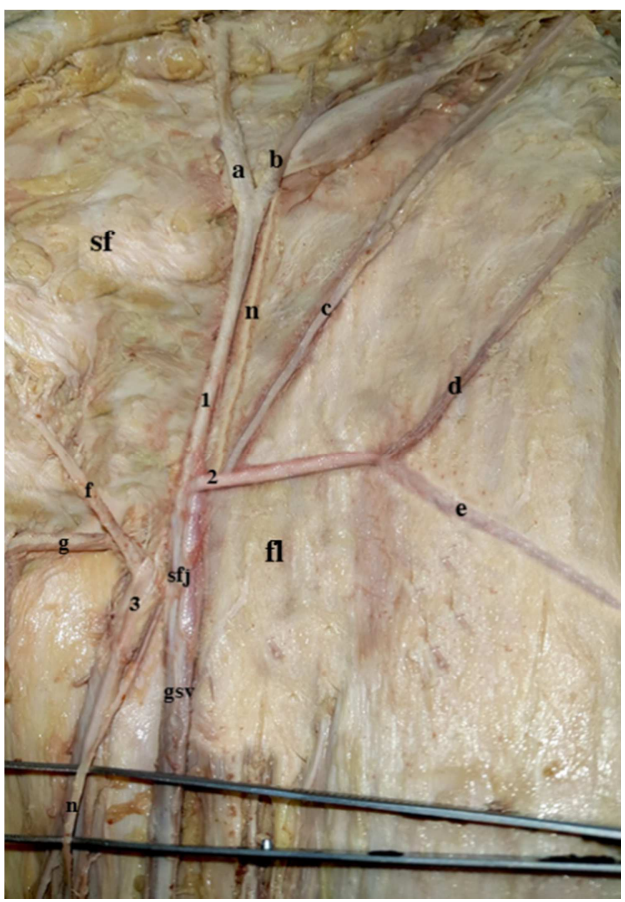

Figure 6. A photograph of left thigh. The gsv is seen inside its fascial canal. There are 3 sfj tributaries (1-3). Tributary 1 is formed by a,b. Tributary 2 is formed by c,d,e. Tributary 3 is formed by f,g. A cutaneous branch (n) from femoral nerve passes behind sfj. The tributaries are superficial to saphenous fascia (sf) and fascia lata proper (fl) through their entire length. The superficial epigastric is (a), the superficial circumflex iliac is (b), while the superficial external pudendal is $(f)$ and the deep external pudendal is $(g)$. 


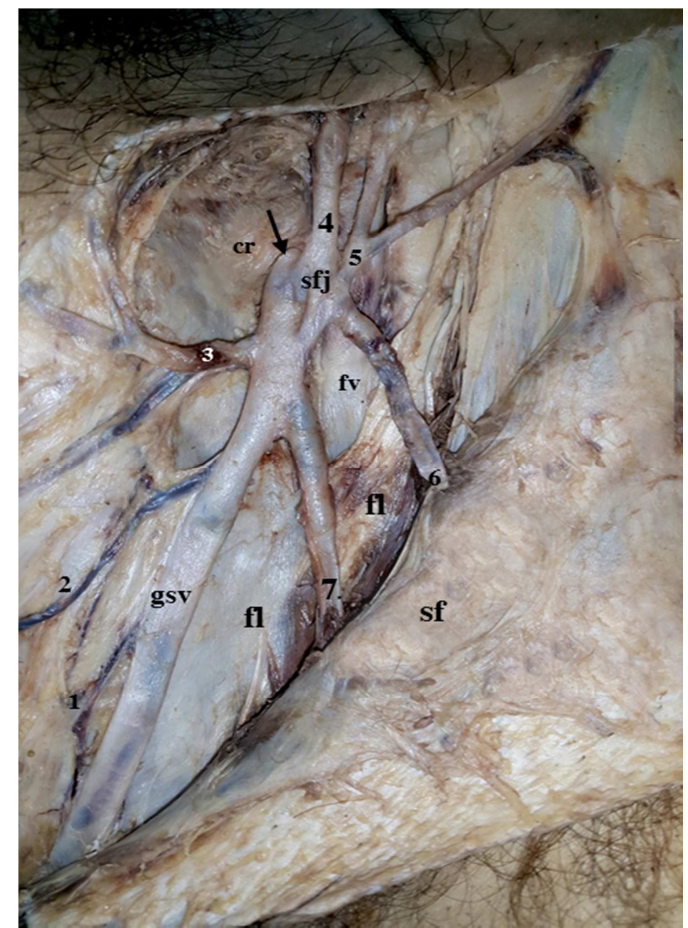

Figure 7. A photograph of the left thigh. The gsv is joined by 7 tributaries (1-7). It passes through the saphenous opening (arrow) in the cribriform fascia (cr) to join the femoral vein ( $f v)$ at the sfj. The proximal part of the tributaries 6, 7 run in the saphenous compartment superficial to fascia lata (fl) and deep to saphenous fascia (sf). The fv is deep to fl. The superficial circumflex iliac is (5), superficial epigastric is (4) while superficial external pudendal is (3).

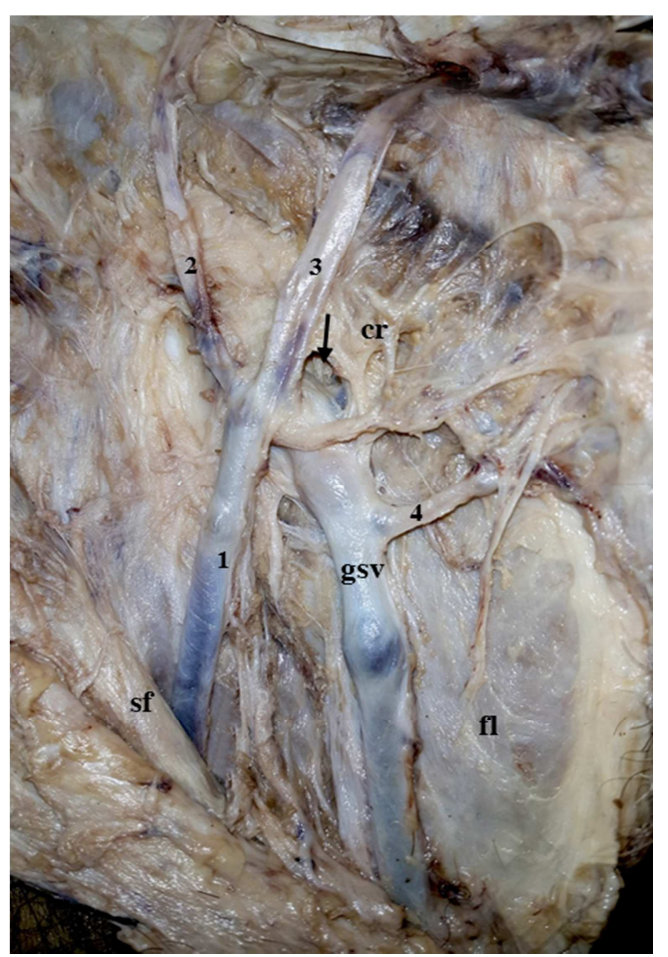

Figure 8. A photograph of right thigh. The gsv is joined by veins (1-4). It passes through the saphenous opening (arrow) in the cribriform fascia (cr). The saphenous fascia (sf) and fascia lata (fl) are noted. The superficial circumflex iliac is (2), superficial epigastric is (3) while superficial external pudendal is (4).

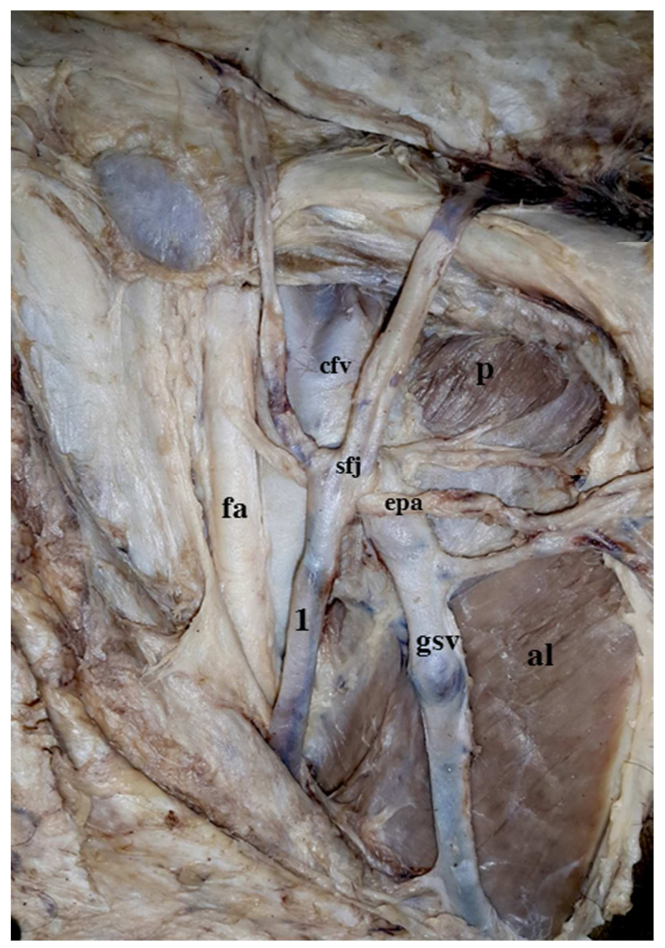

Figure 9. A photograph of the previous specimen after deeper dissection. The sfj and the common femoral vein (cfv) are noted. The external pudendal artery (epa) is noted taking origin from the femoral artery (fa). It passes behind the anterolateral thigh vein (1) in front of gsv below sfj. Adductor longus (al) and pectineus muscles are noted.

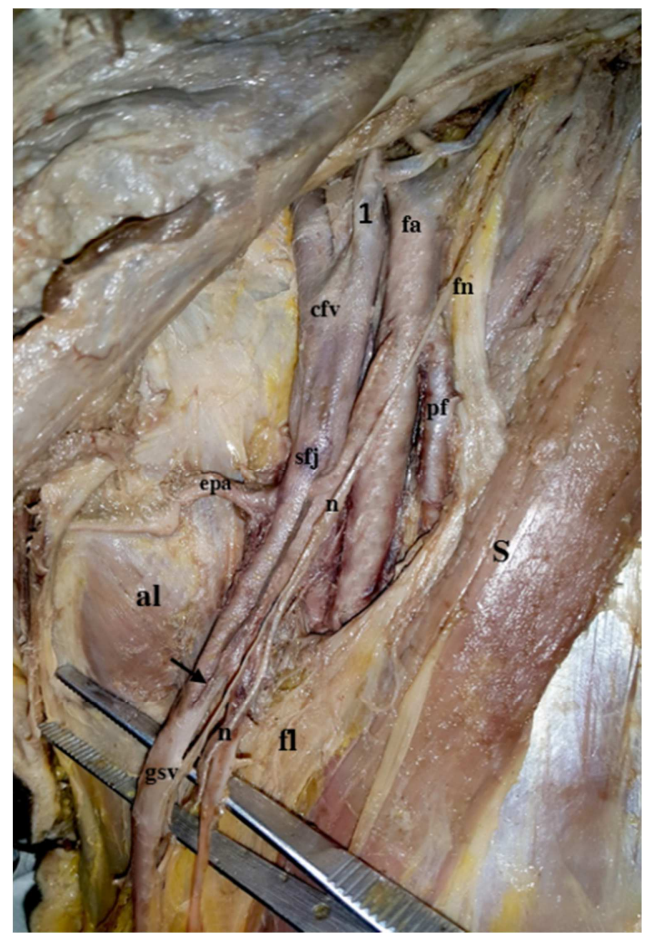

Figure 10. A photograph of left thigh. The gsv joins the cfv at sfj. The cutaneous branch (n) from the femoral nerve (fn) passes just lateral to sfj. Its branches are adherent to the gsv. The femoral artery ( $\mathrm{fa}$ ) and its profunda femoris branch (pf) are noted. The fascia lata (fl) is attached at medial border of sartorius (s). The superficial circumflex iliac vein (1) joins the cfv. The terminal part of gsv is bifid (arrow). The external pudendal artery (epa) passes between the 2 limbs of the bifid gsv just below sfj. The epa is covered by fat in front of adductor longus (al). 


\section{Discussion}

\subsection{Fascial Saphenous Compartment}

The sfj is indeed a complex structure. It is complex not only due to the variable tributaries joining it, but also due to its relation to the deep fascia of the front of the thigh.

The study of the anatomy of the saphenous compartment and its contents can help us to a great extent to understand pathogenesis of lower limb varicose veins and to prevent and treat them in the best way.

The presence of the gsv in the saphenous compartment is a huge dynamic factor aiding venous return from the lower limb and preventing development of varicosities. However, the presence of its tributaries outside the compartment as they drain superficial cutaneous areas can lead to varicosities of these veins.

In the present study, the proximal part of the gsv was in the saphenous compartment in all cases. Superficial tributaries passed through the saphenous fascia and entered the saphenous compartment to join sfj. However, the tributaries were in the saphenous compartment for variable distances. Of course, the longer the distance the tributaries run in the compartment, the less the possibility of developing varicose veins and vice versa.

Type 1 cases $(30 \%)$ are the least expected to develop varicose veins because there is a large triangular fascial saphenous compartment accommodating all superficial tributaries between the 2 layers of deep fascia. Type 2 cases $(30 \%)$ are the most expected to develop varicose veins because all superficial tributaries are outside the fascial canal. Type 3 cases $(40 \%)$ may or may not develop varicosities pending other predisposing factors.

Another factor is the toughness of saphenous fascia. This is very important in preventing varicosities. Saphenous fascia is thicker in females but mainly formed of fatty tissues while in males it is thinner but mainly formed of tough fibrous tissues. Strength of saphenous fascia is crucial in preventing dilatation of veins and development of varicosities.

Papadopoulos et al [10] were the first to describe the fascial canal of the gsv. They described 2 layers of fascia derived from the deep fascia of thigh forming the canal. They noticed tributaries piercing the superficial layer of fascia to enter the canal and join the gsv. They reported that the canal was absent in the thigh in $15 \%$ of cases. However, fascial saphenous compartment was found in all cases of the present study and a triangular saphenous compartment, not just a canal, was recognized in $30 \%$ of cases.

Chen and Prasad [6] recognized the saphenous compartment by ultrasonography. They reported 4 types of the saphenous compartment according to its contents; 1) Single gsv without tributaries. 2) Single gsv and a large tributary which pierces the saphenous fascia to join the gsv. 3) Single gsv lies proximally while a large tributary lies distally. 4) The gsv and the anterior accessory saphenous vein join to terminate in the sfj.

We can clearly see that the previous description is compatible with types 2 and 3 of the present cases but lacks type 1 . Ultrasonography is developing very rapidly and it will not be long before appropriate imaging windows are available to overcome tissue depth in the thigh and detect all types of fascial compartments and thereby predicting who is most likely to develop varicose veins.

\subsection{Variations of Veins}

A comparison between previous studies describing variations of the sfj and the present one is presented in Table 3. In spite of the smaller number of cases in the present study, the results were very close denoting that sfj tributaries were 3-5 in most of cases. Though the number of tributaries is similar in different studies, the precise location of tributaries in relation to gsv, sfj, cfv and saphenous fascia is very variable.

In this study, the most consistent gsv tributary was the superficial external pudendal vein in $90 \%$ of cases. While in the study of Souroullas et al [3], the most consistent gsv tributary was the superficial circumflex iliac vein, which was present in $94.2 \%$. Hemmati et al [11] reported that the mean number of tributaries was $3.87 \pm 0.99$ and the most common tributary was the superficial external pudendal vein in $96.4 \%$.

There are no specific sfj tributaries patterns. The identity of individual tributaries is not that important. What really matters is the location of these tributaries and whether they are mainly inside the saphenous compartment or outside it.

Souroullas et al [3] reported that $40.7 \%$ of cases had $1-3$ direct $\mathrm{cfv}$ tributaries. It was the deep external pudendal vein in $39.5 \%$ of their cases. Donnelly et al [4] reported that $33.4 \%$ of cases had one or more direct cfv tributaries. In the present study, one cfv tributary was found in $30 \%$ of cases. It was the superficial circumflex iliac vein in $25 \%$ of cases. Again, what is important here is not the name of the tributary, but the need to close fascia lata proper after ligation of that tributary in varicose vein surgery to prevent neovascular recurrence.

Although the incidence of bifid gsv was reported to be as high as $24 \%$ by Mansberger et al. [12], $18.1 \%$ by Donnelly et al [4], it was found in $2.3 \%$ by Souroullas et al [3], 5.7\% by Tavlasoglu et al [13] and in $7.5 \%$ of the present series.

Accurate knowledge of the position of the epa is important to avoid its injury. The epa commonly passes between the gsv and the sfj and is often positioned medial to the cfv. It has been suggested that the epa could be an anatomical landmark for identifying the sfj during surgery [14].

Given the considerable variations in gsv, sfj and epa anatomy, reliance on epa to identify sfj could be misleading. On the contrary, the identification of the epa within the operative field could mean the presence of clinically important anatomical variations of the sfj [4].

Murakami et al [15] confirmed that the gsv had an intimate relationship with cutaneous nerves of various origins in the thigh region. They recommend Limited extraction of the gsv in varicose vein surgery in such cases to reduce the risk of nerve injury during the stripping operation. Cutaneous branches of the femoral nerve were found in close proximity to the gsv and sfj in more than $50 \%$ of the present cases. 
Cappelli et al [16] reported that sfj is a multi-structure. Its integrity relies on the competence of the terminal and preterminal valves. Loss of function at these valves is associated with development of varicose veins.

Dickson et al [17] assessed the terminal and preterminal valves of gsv and their distance from the sfj. They reported that the greater the distance to the terminal and preterminal valves, the greater the number of tributaries between them and sfj and the greater the possibility of developing varicose veins.

Classic varicose vein surgery has been challenged. It is complicated by recurrences at the sfj due to overlooked tributaries or development of neovascularization. Recurrent venous reflux occurs in up to $60 \%$ of limbs in patients surviving for 30 years after the 1ry operation [18].

Carandina et al [19] reported that the haemodynamic effect of each part of the sfj has led to new approaches for varicose veins therapy, which are focused on treating the dysfunctional component and sparing those that are not. These approaches include endovenous laser therapy and ultrasound guided foam sclerotherapy.

One of the most common causes of early recurrence following endovenous laser therapy is from neoreflux in nonablated groin tributaries [20].

De Maeseneer et al [21] suggested a potential benefit of closure of the cribriform fascia after sfj ligation to prevent neovascularisation and reduce the incidence of recurrence. They stated that the use of fascia had better results and fewer complications when compared to the use of synthetic patches.

So in terms of neovascular recurrences, it does not matter whether surgeon goes with standard surgery or new approaches. The effectiveness of therapy is dependent on his knowledge of the anatomical variability of sfj. If clinically important variations are overlooked, surgical or less invasive procedures might result in recurrence of varicosities.

\section{Conclusion}

The present work concludes that surgeon's knowledge of anatomical variations at $\mathrm{sfj}$ is essential for better results and less recurrence rates.

More importantly, there are 2 essential hemodynamic factors responsible for antegrade flow through gsv and sfj namely; 1) The presence of $\mathrm{sfj}$ tributaries inside the saphenous compartment and the strength of saphenous fascia. 2) The integrity and position of the terminal and preterminal valves of the gsv.

Respecting fascial layers at the sfj is strongly recommended. Closure of cribriform fascia is not enough. The deep fascia of the front of the thigh should be incised and closed in layers starting with saphenous fascia, then cribriform fascia. Finally, fascia lata proper should be closed if incised to ligate cfv tributaries. This is essential to preserve hemodynamics in the saphenous compartment and prevent neovascular recurrences.

\section{Disclosure of Interests}

The authors declare that there is no conflict of interest.

\section{References}

[1] Gabella G. Cardiovascular system. In Williams PL, editor. Gray's Anatomy. $38^{\text {th }}$ ed. New York: Churchill Livingstone. 1995; p 1596.

[2] Sinnatamby CS, Last RJ. Last's Anatomy: Regional and Applied. $10^{\text {th }}$ ed. Edinburgh: Churchill Livingstone. 2000; p112.

[3] Souroullas P, Barnes R, Smith G, Nandhra S, Carradice D, Chetter I. The classic saphenofemoral junction and its anatomical variations. Phlebology 2017; 32(3): 172-178.

[4] Donnelly M, Tierney S and Feeley TM. Anatomical variation at the saphenofemoral junction. Br J Surg. 2005; 92: 322-325.

[5] Williams A. Pelvic girdle and lower limb. In Standring S, editor. Gray's Anatomy, The Anatomical Basis of Clinical Practice. $39^{\text {th }}$ ed. Churchill Livingstone, Edinburgh. 2005; p 1420 .

[6] Chen SSH and Prasad SK. Long saphenous vein and its anatomical variations. AJUM 2009; 12 (1): 28-31.

[7] Leopardi D, Hoggan BL, Fitridge RA, Woodruff PWH, Maddern GJ. Systematic review of treatments for varicose veins. Ann Vasc Surg. 2009;23:264-276.

[8] Beale RJ, Gough MJ. Treatment options for primary varicose veins-a review. Eur J Vasc Endovasc Surg. 2005;30:83-95.

[9] Kirkpatrick L. A, Feeney B. C. A Simple Guide to IBM SPSS Statistics for Version 20.0, Cengage Learning, Belmont, Calif, USA. 2013.

[10] Papadopoulos NJ, Sherif MF, Albert EN. A fascial canal for the great saphenous vein: gross and microanatomical observations. J Anat.1981; 132 (3): 321-329.

[11] Hemmati H, Baghi I, Zadeh KT, Okhovatpoor N, Nejad EK. Anatomical variations of the saphenofemoral junction in patients with varicose veins. Acta Medica Iranica. 2012; 50 (8): 552-555.

[12] Mansberger AR, Yeagher GH, Smelser RM, Brumback FM. Saphenofemoral junction anomalies. Surg Gynecol Obstet. 1950;91:533-536.

[13] Tavlasoglu MG, Guler A, Gubuz HA, Tanriseven M, Kurkluoglu M, Yesil FG. Anatomical variations of the saphenofemoral junction encountered during venous surgery. J Cardiovasc Surg. 2013; 1: 5-7.

[14] Cushieri A, Steele RJC and Moossa AR. Essential surgical practice: higher surgical training in general surgery, 4th ed. London: Arnold. 2002.

[15] Murakami G, Negishi N, Tanaka K. Anatomical relationship between saphenous vein and cutaneous nerves. Okajimas Folia Anat Jap. 1994; 71(1):21-23.

[16] Cappelli M, Molino Lova R, Ermini S, Zamboni P. Hemodynamics of the sapheno-femoral junction. Patterns of reflux and their clinical implications. Int Angiol. 2004;23:25-8. 
[17] Dickson R, Hill G, Thomson IA, van Rij AM. The valves and tributary veins of the saphenofemoral junction: ultrasound findings in normal limbs. Veins and Lymphatics 2013; 2(18):63-67.

[18] Fischer R, Chandler JG, De Maeseneer MG. The unresolved problem of recurrent saphenofemoral reflux. JACS 2002; 195 (1): 80-94.

[19] Carandina S, Mari C, De Palma M, Marcellino MG, Cisno C, Legnaro A, et al. Varicose vein stripping vs haemodynamic correction (CHIVA): a long term randomised trial. Eur J Vasc Endovasc Surg. 2008;35:230-237.
[20] Brittenden J, Cotton SC, Elders A, Ramsay CR, Norrie J, Burr $\mathrm{J}$, et al. A randomized trial comparing treatments for varicose veins. New Engl J Med. 2014; 371: 1218-1227.

[21] De Maeseneer MG, Philipsen TE, Vandenbroeck CP, Lauwers PR, Hendriks JM, De Hert SG, et al. Closure of the cribriform fascia: an efficient anatomical barrier against postoperative neovascularisation at the saphenofemoral junction? a prospective study. Eur J Vasc Endovasc Surg. 2007;34, 361366. 